\title{
Gollop-Wolfgang Complex: Clinical and Imaging Implications
}

\author{
Robert Hunter Vanderberg ${ }^{1}$ Thomas Block $^{1} \quad$ Thomas Gates $^{1} \quad$ Juan Gomez ${ }^{1}$ \\ ${ }^{1}$ Department of Radiology, Louisiana State University Health Science \\ Center, Shreveport, Louisiana, United States \\ Address for correspondence Robert Hunter Vanderberg, MD, 1501 \\ Kings Highway, Shreveport, LA 71103, United States \\ (e-mail: rvand5@lsuhsc.edu).
}

Indian J Radiol Imaging 2021;31:721-724.

\author{
Abstract \\ Keywords \\ - bifid femur \\ - ectrodactyly \\ - Gollop-Wolfgang \\ complex \\ - hemimelia
}

Gollop-Wolfgang complex is defined as the presence of a distal bifid femur and tibial hemimelia with or without hand ectrodactyly. The condition commonly presents with several skeletal abnormalities and internal organ congenital defects. We hereby report a case with a classical presentation of Gollop-Wolfgang complex.

\section{Introduction}

Gollop-Wolfgang complex is defined as the presence of a distal bifid femur and tibial hemimelia with or without hand ectrodactyly. ${ }^{1-6}$ Most cases of Gollop-Wolfgang complex are accompanied by other congenital defects, such as the VACTERL (vertebral anomalies, anal atresia, cardiovascular anomaliesmost commonly ventricular septal defect [VSD], tracheoesophageal fistula, esophageal atresia, renal anomalies, and limb defects - most commonly radial ray defects) sequence. ${ }^{4-8}$

According to the U.S. Office of Rare Diseases of the National Institute of Health, there have been only 200 cases reported until now. ${ }^{1}$ Gollop-Wolfgang complex is documented to have an incidence of $\sim 1: 1,000,000$ live births. ${ }^{5,7,9}$ The etiology of Gollop-Wolfgang complex is postulated to be an error in the complex genetic control of limb development, but the exact cause remains unclear. ${ }^{1-3}$ The mode of inheritance is autosomal dominant with reduced penetrance and variable expressivity; however, neomutations cannot be excluded. ${ }^{7,9}$

\section{Case Presentation Report}

A 6-month-old African American girl born at 38 weeks by cesarean section to a 22-year-old African American mother presented at birth, with multiple congenital anomalies including a single umbilical artery, polyhydramnios, hemivertebrae, several appendicular, and skeletal deformities (absence of both radii and tibias). Atrial septal defect (ASD), VSD, and patent ductus arteriosus (PDA) had also been identified on antenatal ultrasound. The mother followed routine prenatal care, including counseling for pregnancy termination in another institution, and also consumed prenatal vitamins. She denied any symptoms or complications during the pregnancy other than the aforementioned congenital abnormalities found on the 20-week anatomy ultrasound. She also denied a family history of congenital abnormalities, limb deficiencies, or teratogenic substance use.

At delivery, the newborn's physical examination was remarkable for cyanosis, a heart murmur, several limb deformities including bilateral talipes equinovarus, ectrodactyly, and protrusion of the distal femur anteromedially on the left knee joint.

The radiographs obtained at birth confirmed findings consistent with Gollop-Wolfgang complex and a class 3 tracheoesophageal fistula. Initial chest X-ray (-Fig. 1) showed an orogastric tube with its tip projecting at the level of the clavicles, with gaseous distention of the stomach and proximal small bowel, all suspicious for esophageal atresia
DOI https://doi.org/ 10.1055/s-0041-1736396. ISSN 0971-3026.

\footnotetext{
(C) 2021. Indian Radiological Association. All rights reserved. This is an open access article published by Thieme under the terms of the Creative Commons Attribution-NonDerivative-NonCommercial-License, permitting copying and reproduction so long as the original work is given appropriate credit. Contents may not be used for commercial purposes, or adapted, remixed, transformed or built upon. (https://creativecommons.org/ licenses/by-nc-nd/4.0/) Thieme Medical and Scientific Publishers Pvt. Ltd., A-12, 2nd Floor, Sector 2, Noida-201301 UP, India
} 


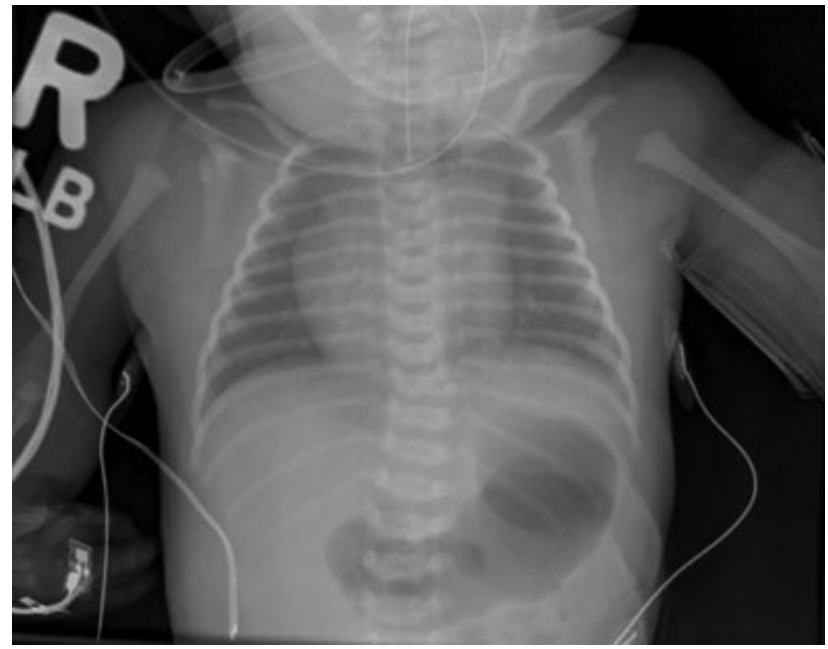

Fig. 1 Initial anteroposterior view of the chest. Orogastric tube is visualized at the level of the clavicles, there is gaseous distention of the stomach consistent with esophageal atresia plus distal tracheoesophageal fistula. A widened cardiothymic silhouette is also seen suggesting a congenital cardiac defect.

with tracheoesophageal fistula. Prominent cardiothymic silhouette, diffuse mild bilateral granular opacities, fusion of the first and second right ribs, 13 pairs of ribs, and a vertebral anomaly involving the left aspect of the T-10 vertebrae were also noted. X-rays of both upper extremities (-Figs. 2 and $\mathbf{3}$ ) demonstrated the absence of the radii and thumbs, significant angulation at the wrists with respect to the normal ulna, deformed hands that were deviated proximally, medially, and anteriorly. X-ray of the right lower extremity ( - Fig. 4) revealed a grossly normal femur, absence of one of the leg bones (difficult to determine the specific bone due to the projection and abnormal appearance of the remaining bone), abnormal alignment of the knees, four metatarsals and four digits, abnormal relationship of the leg with respect to the ankle and foot, and soft tissue

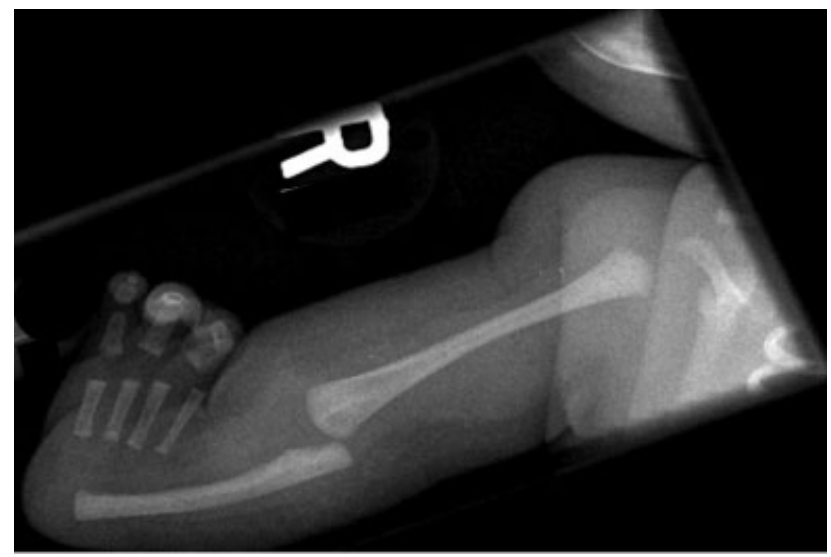

Fig. 2 Anteroposterior view of the right upper extremity. The absence of radius and thumb leads to initially possible differentials diagnoses of thrombocytopenia-absent radius syndrome or Fanconi anemia which were dismissed secondary to normal complete blood count. Angulation of the wrist joint and abnormalities of the metacarpal and digital bones are also evident.

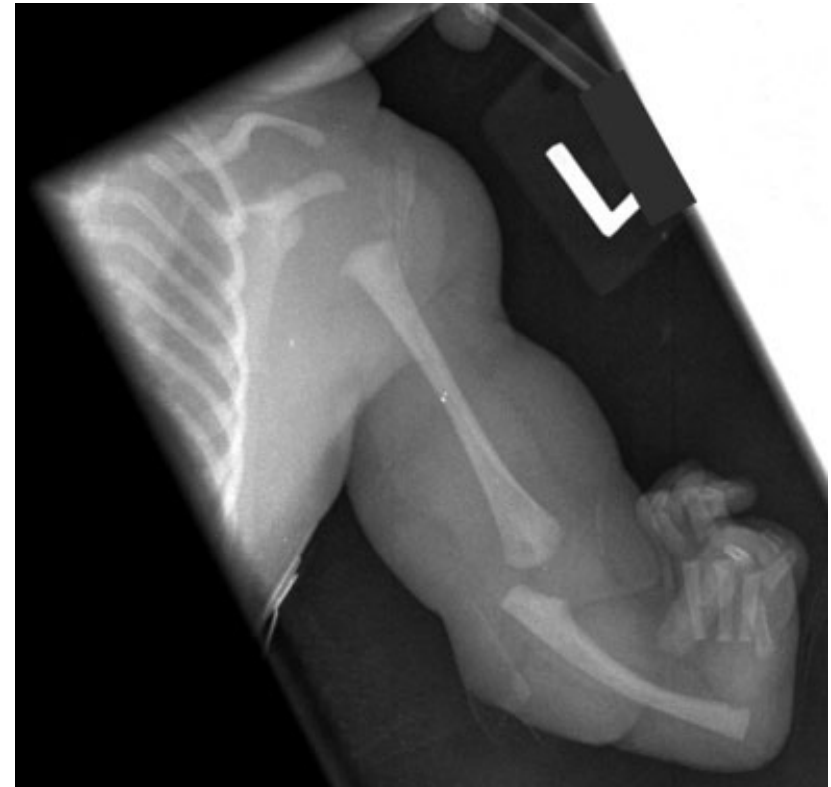

Fig. 3 Anteroposterior view of the left upper extremity demonstrates the absence of radius and thumb, significant angulation at the wrists with respect to the normal ulna, and deformed hand that was deviated proximally, medially, and anteriorly.

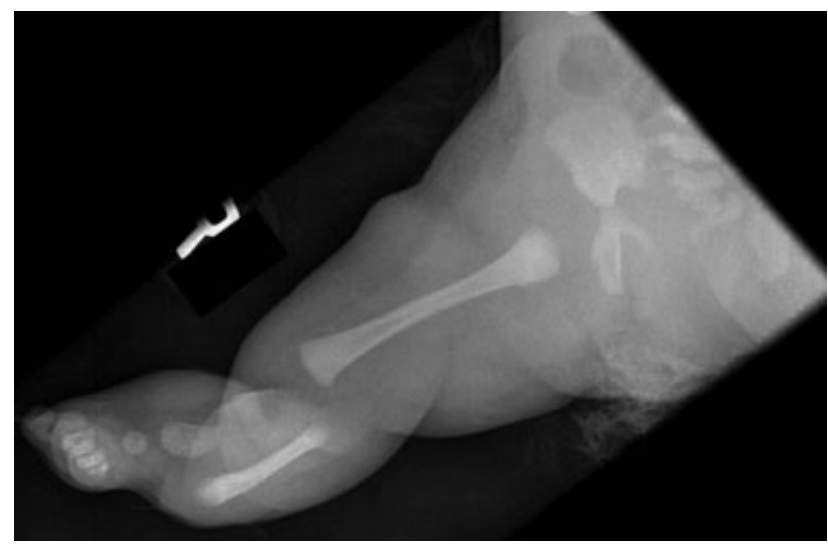

Fig. 4 Anteroposterior (AP) view of the right lower extremity. Normal development of the femur is evident; however, there is only one bone present in the leg. Viewing both AP and frog-leg lateral (not shown) views, it was difficult to determine if this leg bone was the tibia or fibula. Talipes equinovarus and bony foot abnormalities are also present.

deformity. X-rays of the left lower extremity (-Fig. 5) revealed a normal proximal femur but a bifid distal femur, absence of one leg bone as seen on the opposite leg, three metatarsal bones, and abnormal relationship at the ankle with respect to the leg. The patient underwent several laboratory and genetic testings. Karyotyping showed 46 $X X$. A complete blood count with differential was performed, which was reported to have a normal range values, thus making Fanconi anemia or thrombocytopenia with absent radius syndrome unlikely. The patient also underwent successful tracheoesophageal fistula surgery confirmed by follow-up barium swallow studies. 


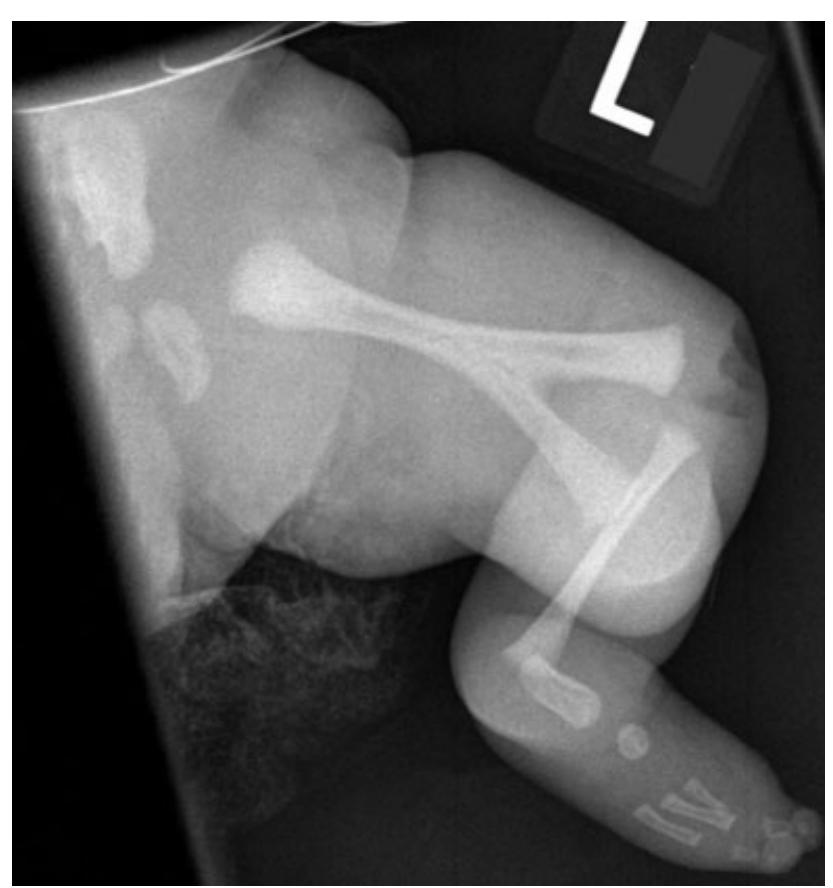

Fig. 5 Frog-leg lateral view of the left lower extremity reveals distal bifid femur, presence of only one leg bone, three metatarsal bones, and abnormal relationship at the ankle with respect to the leg.

\section{Discussion}

Gollop-Wolfgang complex belongs to the skeletal dysplastic classification of dysostoses, which refers to malformations or absence of individual bones singly or in combination. According to the theory of Lewin and Opitz, the growth of the lower limb is under the control of two developmental fields, the tibia and fibula. The tibial developmental field controls the development of the distal femur, tibia, and hallux. Thus, a defect in this field results in distal femur duplication, tibia agenesis, and preaxial polydactyly or ectrodactyly. The fibula developmental field controls the development of the fibula, lateral rays of the foot, lateral knee ligaments, proximal femur, acetabulum, and pubic bones. A defect in this field results in fibular hypoplasia, ectrodactyly, proximal focal femoral deficiency, and deficiency of lateral knee ligament. There is a strong association between the development of the fibula and tibial fields. 1,7,10

Findings of Gollop-Wolfgang complex in antenatal ultrasound include increased nuchal translucency between 11 and 14 weeks of gestation and tibial agenesis with femoral bifurcation, clubfoot, contracture of the knees, and agenesis of the metacarpal bones at 20 weeks. Amniocentesis can be performed for chromosomopathies, detection of chromosomal abnormalities, and genetic counseling. ${ }^{4,11,12}$ Prenatal exposure to valproic acid is associated with an increased risk of congenital malformations, especially limb defects such as radial ray defects. ${ }^{7}$

Regarding the differential diagnosis for this disease, Forzano et al stated that the tibial agenesis-ectrodactyly syndrome is the only condition that presents a significant overlap with Gollop-Wolfgang complex. ${ }^{12}$ Additional anomalies that should be excluded for the diagnosis of Gollop-Wolfgang complex are cleft palate, cleft lip, and ocular abnormalities. In our case, the presence of a distal bifid femur, absent radii, ectrodactyly, ASD, VSD, PDA, and tracheoesophageal fistula leads the pediatric radiology team to a clinical and radiologic diagnosis of Gollop-Wolfgang complex. Information regarding the mother's decision to terminate or not to terminate the pregnancy was not available because mother's routine prenatal care and counseling had been performed in another institution.

There are three classification systems of tibial deficiency: Kalamchi and Dawe, Jones, and the newer Weber classification. Jones classification which is the most commonly utilized has divided it into four types. Type 1: The tibia cannot be seen on radiographs at birth. In subtype $1 \mathrm{a}$, the tibia is completely absent and the ossific nucleus of the distal femoral epiphysis is hypoplastic. In subtype $1 \mathrm{~b}$, the proximal part of tibia is present, but unossified at birth, hence appears absent on plain radiograph. In this type, there is normal ossification of the distal femoral epiphysis. Type 2: The proximal part of the tibia is ossified and visible on radiographs at birth, but the distal tibia is absent. Type 3: The distal part of the tibia is ossified and visible, but the proximal portion of the tibia is absent. This is the least common type of tibia hemimelia. Type 4: The tibia is short, and there is distal tibiofibular diastasis. ${ }^{1,6,7}$

In terms of treatment, prosthetic reconstruction of tibia versus knee disarticulation depends only on the quantum of quadriceps function. ${ }^{13,14}$ According to the Jones classification, our patient would classify as type 1a on the left lower extremity and type $1 \mathrm{~b}$ on the right lower extremity qualifying for a knee disarticulation and realignment of the left lower extremity and tibiofibular synostosis of the right lower extremity.

\section{Conclusion}

There are several findings in both clinical and radiographic presentations between our case and prior documented cases of Gollop-Wolfgang complex. Regarding the etiology of this disease, we support the theory proposed by Lewin and Opitz of lack of tibial developmental fields causing defects in distal femur development. ${ }^{15}$ Although our patient's condition was most likely spontaneous due to the absence of risk factors, we also support the theories of possible associations between genetic and environmental factors. Imaging studies such as antenatal ultrasound and postnatal radiographs contribute to the early diagnosis of this condition. Radiographs also assist in the classification of tibial deficiency, providing beneficial information for the treatment.

\section{Declaration of Patient Consent}

The authors have obtained the parents' (mother's) permission for publication of the case in a scientific journal, with an undertaking for all efforts to conceal the patient's identity.

\section{Financial Support and Sponsorship}

None. 
724 Gallop-Wolfgang Complex: Must Not Forget Vanderberg et al.

\section{Source(s) of Support}

None.

\author{
Presentation at a Meeting \\ None.
}

\section{Conflict of Interest}

None declared.

\section{References}

1 Ondari J, Kinyanjui J, Miano P, Sang E, Oburu E, Maru M. Femoral bifurcation and bilateral tibial hemimelia: case report. Pan Afr Med J 2018;30:99

2 Habou O, Magagi IA, Adamou H. Gollop-Wolfgang complex. J Neonatal Surg 2017;6(01):19

3 Nagata E, Kano $\mathrm{H}$, Kato $\mathrm{F}$, et al. Japanese founder duplications/triplications involving BHLHA9 are associated with or without long bone deficiency and Gollop-Wolfgang complex. Orphanet J Rare Dis 2014;9:125

4 Caforio L, Pagnotta G, Romiti A, Familiari A, Donati F, Bagolan P. Prenatal diagnosis of Gollop-Wolfgang complex. Ultrasound Obstet Gynecol 2015;45(04):488-490

5 Qureshi IA, Gudepu RK, Chava R, et al. Case report: GollopWolfgang complex in a 5 month old baby. F1000 Res 2014;3:315

6 Wada A, Nakamura T, Fujii T, et al. Limb salvage treatment for Gollop-Wolfgang complex (femoral bifurcation, complete tibial hemimelia, and hand ectrodactyly). J Pediatr Orthop B 2013;22 (05):457-463

7 Alessandri JL, Isidor B, David A, et al. Tibial developmental field defect in valproic acid embryopathy: report on three cases. Am J Med Genet A 2010;152A(11):2805-2809

8 Erickson RP. Agenesis of tibia with bifid femur, congenital heart disease, and cleft lip with cleft palate or tracheoesophageal fistula: possible variants of Gollop-Wolfgang complex. Am J Med Genet A 2005;134(03):315-317

9 Pandy D, Pal MV, Nambiar J, Lewis L, Pandy V. Gollop-Wolfgang complex-a rare limb deficiency syndrome: case report and review of literature. Internet J Gynecol Obstet 2007;9:1-5

10 Pavone L, Viljoen D, Ardito S, et al. Two rare developmental defects of the lower limbs with confirmation of the Lewin and Opitz hypothesis on the fibular and tibial developmental fields. Am J Med Genet 1989;33(02):161-164

11 Mendilcioglu I, Mihci E, Pestereli E, Simsek M. Prenatal diagnosis of Gollop-Wolfgang complex (tibial agenesis and femoral bifurcation). Prenat Diagn 2009;29(02):182-186

12 Forzano F, Viassolo V, Castagnetta M, et al. Prenatal diagnosis of Gollop-Wolfgang complex. Prenat Diagn 2009;29(07):724-726

13 Loder RT, Herring JA. Fibular transfer for congenital absence of the tibia: a reassessment. J Pediatr Orthop 1987;7(01):8-13

14 Albright P, Veenstra J, Habeck J, Bovid K. Lower extremity surgical treatment to improve function in a patient with Gollop-Wolfgang complex. JBJS Case Connect 2019;9(02):e0254

15 Lewin SO, Opitz JM. Fibular a/hypoplasia: review and documentation of the fibular developmental field. Am J Med Genet Suppl 1986;2:215-238 